\section{F.-B. CARTIAUX A. GELLÉE}

École nationale des ponts et chaussées 6 et 8 , av. Blaise-Pascal 77455 Marne-la-Vallée Cedex 02

\section{P. DE BUHAN}

G. HASSEN

Institut Navier, ENPC 6 et 8 , av. Blaise-Pascal 77455 Marne-la-Vallée Cedex 02 debuhan@imsgc.enpc.fr

\title{
Modélisation multiphasique appliquée au calcul d'ouvrages en sols renforcés par inclusions rigides
}

Une modélisation des sols renforcés par inclusions rigides, qualifiée de multiphasique, est proposée, permettant de prendre explicitement en compte les phénomènes d'interaction entre le sol et les inclusions, qui gouvernent la façon dont les charges appliquées à Jouvrage se transmettent aux renforcements et conditionnent l'efficacité des ces derniers. On montre en particulier dans cet article, comment l'identification de coefficients relatifs aux interactions latérale et en tête d'inclusion, réalisée à partir de simulations numériques sur une configuration simple de référence, permet la mise en cuvre d'un tel modèle dans le cadre d'un code par éléments finis. L'outil de calcul ainsi mis au point est alors appliqué à I'analyse du tassement d'un sol compressible consécutif à l'édification d'un remblai, et à la réduction considérable de ce tassement pouvant résulter d'une solution de renforcement par inclusions rigides.

Mots-clés: renforcement, inclusions rigides, modèle multiphasique.

\section{A multiphase model for the design of soils reinforced by rigid inclusions}

This paper advocates a so-called multiphase model for soils reinforced by rigid inclusions, aimed at capturing the soilinclusions interaction phenomena, which play a decisive role in the way the applied loads are transferred to the inclusions, and thus clearly affect the performance of the reinforcement scheme. It is shown in particular how the identification of coefficients, relating to the lateral as well as pile head interactions, performed from numerical simulations on a simple reference case, allows to implement such a model in a finite element formulation. The so-obtained numerical code is then applied to the settlement analysis of a soft soil subject to the weight of an embankment, and to the correlative reduction of this settlement which could be expected from the incorporation of rigid inclusions.

Key words : reinforcement, rigid inclusions, multiphase model. 


\section{1}

\section{Introduction}

Le renforcement de sols de fondation de qualité médiocre, en raison notamment de leur grande compressibilité, par des inclusions « rigides ») verticales (parfois désignées dans le cas d'inclusions semi-rigides sous l'appellation de " colonnes à module contrôlé » (CMC) : Liauzu et Pezot, 2001), connait depuis quelques années un essor important (Alexiew et Vogel, 2002 ; Briançon, 2003 ; Briançon et al., 2004 ; Lacazedieu et al., 2004 ; Jenck et al., 2005). Cette technique consiste à transférer la charge due à l'ouvrage placé en surface vers une couche de sol de bien meilleure qualité (substratum), par la combinaison d'un matelas granulaire de répartition placé entre l'ouvrage et la couche de sol compressible, et l'incorporation d'inclusions cylindriques verticales, généralement fabriquées en béton, disposées selon un maillage régulier au sein de la couche compressible, comme schématisé sur la figure 1. Les inclusions peuvent être reliées en tête à une semelle de radier (fondations mixtes), ou bien comme dans l'exemple du procédé de renforcement retenu pour stabiliser les fondations des piles du pont de Rion-Antirion en zone sismique (Pecker, 1998; Pecker et Teyssandier, 1998), être des tubes métalliques «flottants »), c'est-à-dire sans contact avec un substratum.

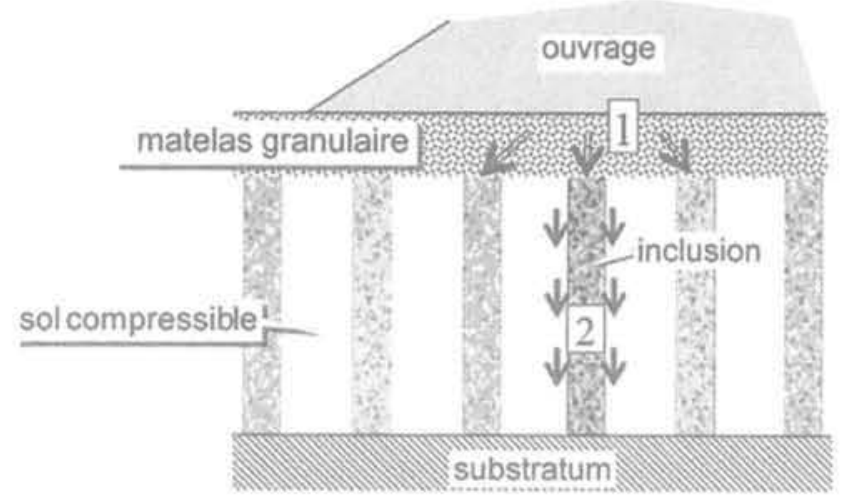

FIG.1 Schéma de principe de la technique de renforcement d'un sol de fondation par inclusions rigides.

Outline of the reinforcement technique of a foundation soil by the incorporation of rigid inclusions:

Sur un plan qualitatif, se limitant au cas d'inclusions en contact avec un substratum rigide, les mécanismes gouvernant un tel report de charge sont de deux types. Par $\alpha$ effet de voûte $n$ tout d'abord au sein du matelas granulaire, une part significative de la charge est reprise par les têtes d'inclusions (1), tandis que par le biais du frottement latéral entre le sol et les inclusions (effet d'u accrochage $)$ ou de frottement négatif (2)), les inclusions viennent progressivement reprendre une part croissante de cette charge, soulageant ainsi d'autant le sol compressible, et réduisant corrélativement dans des proportions importantes le tassement global de la couche de fondation (Combarieu, 1988).

L'analyse et la prise en compte de ces mécanismes d'un point de vue quantitatif est indispensable pour qui veut parvenir à une méthode rationnelle de calcul et de dimensionnement de ce type d'ouvrages, en ce qui concerne notamment la réduction de tassement que l'on est en droit d'attendre de cette technique de renforcement. Elle se révèle néanmoins très complexe, dans la mesure où, se référant par exemple à une méthode de calcul par éléments finis, elle nécessite une discrétisation fine tridimensionnelle des inclusions et du sol environnant, aboutissant ainsi très rapidement à des problèmes insurmontables en raison de la taille numérique du problème, ou tout au moins incompatibles avec l'exigence d'un dimensionnement rapide de l'ouvrage.

Dans le but de surmonter de telles difficultés, la présente contribution propose un modèle de calcul simplifié, qualifié de modèle " multiphasique », qui s'inspire d'une approche par homogénéisation, tout en remédiant aux insuffisances de cette dernière. Ce modèle tire partie de deux caractéristiques majeures de ce type de renforcement:

a) la distribution des inclusions au sein du massif est périodique :

b) l'échelle caractéristique du renforcement (espacement entre deux inclusions voisines par exemple) peut être raisonnablement considérée comme inférieure (mais pas nécessairement très inférieure) aux dimensions d'ensemble de l'ouvrage.

Nous verrons comment l'utilisation d'un tel modèle de calcul, sous réserve d'en avoir préalablement identifié les paramètres constitutifs, conduit alors à une diminution radicale des temps de caicul nécessaires à la simulation du comportement de l'ouvrage, et notamment à l'évaluation de ses tassements, par rapport à une méthode consistant à traiter directement le sol renforcé comme un milieu composite. L'étude est ici conduite dans l'hypothèse où le comportement des différents matériaux (sol, renforcements, matelas granulaire) est supposé élastique linéaire.

\section{2}

\section{L'approche par homogénéisation et ses limites}

On s'intéresse à l'analyse du tassement d'une couche d'épaisseur $\mathrm{H}$, constituée d'un sol homogène élastique, de module d'Young $\mathrm{E}_{\text {, }}$ et coefficient de Poisson $v_{s}$, surmontée d'un matelas d'épaisseur h en matériau granulaire de caractéristiques $E_{q}$ et $v_{u}$, et renforcée par un groupe d'inclusions verticales cylindriques de rayon $p$, disposées selon un maillage périodique carré de côté e, égal à l'entre axe entre les inclusions (Fig. 2). On désigne par $E_{b}$ et $v_{b}$ les caractéristiques élastiques du matériau constitutif des ces inclusions (béton). L'ensemble est soumis à une surcharge uniforme de densité égale à $\mathrm{q}$.

L'application d'une méthode d'homogénéisation classique pour traiter un tel problème consiste à substituer au sol renforcé composite un milieu homogène anisotrope équivalent dont on peut déterminer les coefficients d'élasticité à partir de ceux des constituants (sol et renforcement), ainsi que de la fraction volumique du matériau de renforcement définie par :

$$
\eta=\frac{\pi \rho^{2}}{\mathrm{e}^{2}}
$$

Compte tenu du fait que cette dernière est faible (inférieure à quelques pourcents), tandis que le module du béton constitutif du renforcement est bien plus grand 


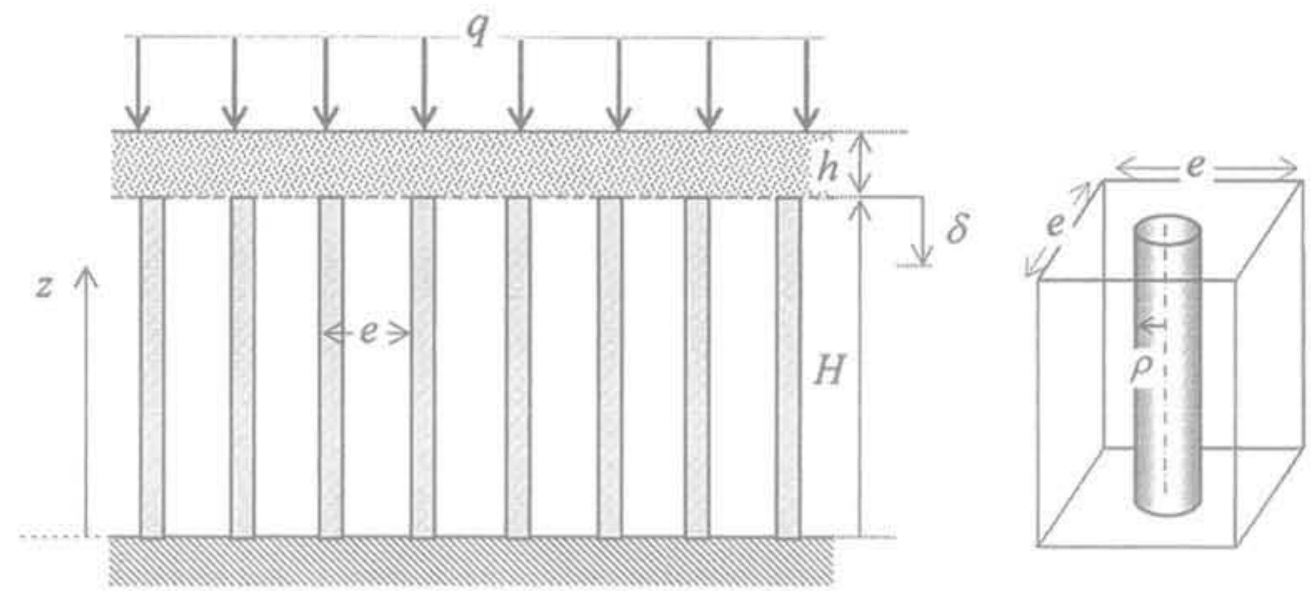

FIG.2 Analyse du tassement sous charge uniforme d'une couche de sol renforcée par une distribution régulière d'inclusions rigídes. Settlement analysis of a reinforced soil layer under uniform surface loading.

que celui du sol $\left(E_{b} \gg E_{s}\right.$ ); on peut montrer (voir par exemple Greuell et al., 1994, ainsi que Bernaud et al., 1995, où cette méthode est appliquée aux tunnels renforcés par boulonnage) que le module oedométrique du sol renforcé dans la direction verticale, c'est-à-dire dans la direction du renforcement, s'écrit tout simplement:

$$
E_{\text {hom }}^{\text {oed }}=E_{\mathrm{s}}^{\text {oed }}+\eta E_{\mathrm{b}}
$$

où $E_{s}^{o e d}$ est le module oedométrique du sol en l'absence de renforcement, défini par :

$$
E_{s}^{\text {oed }}=E_{s} \frac{\left(1-v_{s}\right)}{\left(1+v_{s}\right)\left(1-2 v_{s}\right)}
$$

Dans ces conditions il est facile de voir que, sous l'action de la surcharge $q$, le tassement en surface $(\mathrm{z}=\mathrm{H})$ de la couche de sol non renforcée est tout simplement :

$$
\delta_{0}=H \frac{q}{E_{s}^{\text {oed }}}
$$

tandis que celui de la couche renforcée s'écrit:

$$
\delta=H \frac{q}{E_{\text {bor }}^{\text {oed }}}=\frac{\delta_{0}}{\left(1+\eta E_{b} / E_{s}^{\text {oed }}\right)}
$$

Cette méthode permet en outre d'accéder à la proportion du chargement qui est reprise par les inclusions de renforcement. En effet la contrainte verticale totale (ou macroscopique) peut s'écrire ${ }^{(1)}$ :

$$
\Sigma_{z z}=-\mathrm{q}=-E_{\text {hom }}^{\text {oed }} \frac{\delta}{\mathrm{H}}=-\underbrace{\mathrm{E}_{\mathrm{s}}^{\mathrm{oed}} \frac{\delta}{\mathrm{H}}}_{\mathrm{q}_{5}}-\underbrace{\eta E_{\mathrm{b}} \frac{\delta}{\mathrm{H}}}_{\mathrm{q}_{z}}
$$

où le terme $q$, représente l'effort de compression repris par les inclusions de renforcement calculé par unité de surface transversale, puisque :

$$
q_{r}=\frac{\left(\pi \rho^{2}\right) E_{b}(\delta / H)}{e^{2}}=\frac{N_{r}}{e^{2}}
$$

Désignant par $\lambda=q_{r} / q$ la fraction du chargement repris par les inclusions de renforcement, que nous appellerons facteur de report de charge, l'application de la méthode d'homogénéisation conduit en définitive l'évaluation suivante de ce facteur sans dimension:

$$
\lambda=\frac{\eta E_{\mathrm{b}}}{E_{\text {hom }}^{\text {oed }}}=\frac{1}{1+E_{\mathrm{s}}^{\text {oed }} / \eta E_{\mathrm{b}}}
$$

(1) On adopte dans tout cet article la convention des contraintes de traction comptées positivement.
Un calcul préalable (Rospars ét al., 2005) permet de mettre immédiatement en évidence les limites d'une telle méthode d'homogénéisation, grâce à une comparaison effectuée entre le résultat (8) fourni par cette méthode et celui obtenu par une simulation par éléments finis. Compte tenu de l'uniformité de la charge appliquée en surface et de la répartition périodique des renforcements au sein du massif de sol, la résolution de ce problème par cette dernière approche se ramène à celle du problème auxiliaire, relatif à une cellule élémentaire de section carrée avec les conditions aux limites appropriées, de type oedométrique (Fig. 3, sur laquelle est indiqué le jeu de données utilisé dans les calculs). La partie droite de la figure 3 montre bien que, tandis que la méthode d'homogénéisation donne un facteur de report de charge uniforme sur toute la longueur des inclusions, égal à $\lambda \cong 0,99$ (trait plein vertical sur le graphique de la figure), l'évaluation par voie numérique (symboles de forme carrée sur le graphique) montre que ce facteur de charge décroit rapidement à mesure que l'on s'approche des têtes d'inclusions, où il ne vaut plus que $\lambda \cong 0,27$.

\section{3}

\section{Le sol renforcé vu sous l'angle d'un milieu biphasique}

L'objet de la modélisation multiphasique (en l'occurrence ici biphasique) du sol renforcé est précisément de continuer à tirer partie d'une approche de type homogénéisation, tout en remédiant aux insuffisances de cette dernière que nous venons de mettre en lumière. Nous nous limiterons ici à une présentation simplifiée de ce modèle, adaptée à notre problème spécifique, en renvoyant le lecteur intéressé par un exposé plus détaillé, relatif notamment à la construction de ce modèle, à de Buhan et Sudret (2000), Sudret et de Buhan (2001), ainsi que Bennis et de Buhan (2003) ou encore tout récemment Hassen et de Buhan (2005, 2006).

L'idée intuitive qui préside à l'élaboration d'une telle modélisation est double :

- substituer au sol renforcé composite, non pas un seul comme dans la méthode d'homogénéisation, mais deux milieux continus homogènes superposés géométriquement, en interaction mutuelle, appelés « phases »), qui représentent respectivement le sol et le réseau d'inclusions de renforcement à l'échelle macroscopique (Fig. 4) ; 

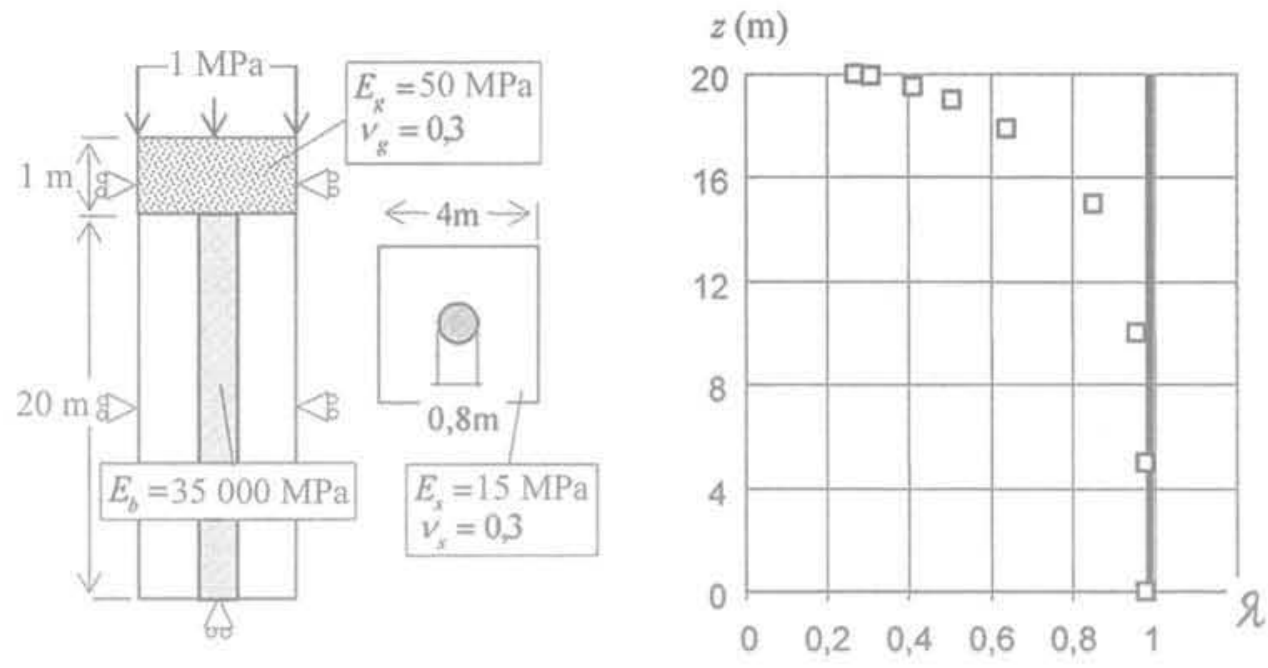

FIG. 3 Comparaison des facteurs de report de charge calculés respectivement par la méthode d'homogénéisation et la méthode des éléments finis (Rospars et al., 2005).

Homogenization vs finite element simulations of the inclusion load carrying capacity.

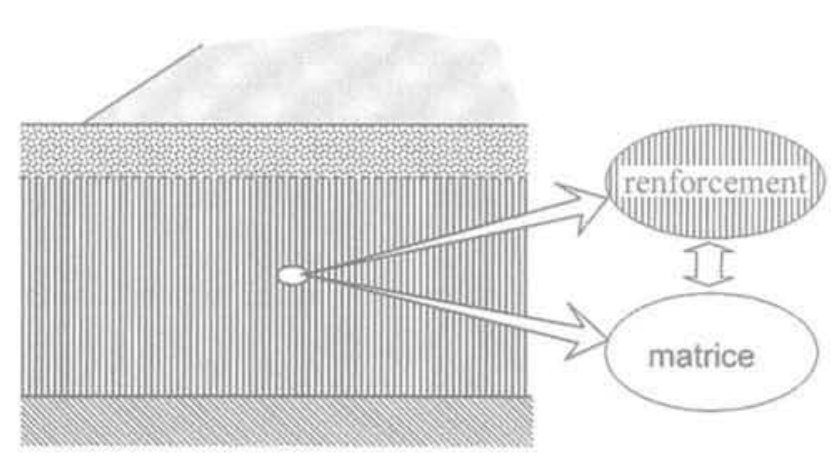

FG. 4 Principe de la modélisation biphasique du sol renforcé.

Principle of the multiphase approach applied to reinforced soils.

- prendre en compte le fait que la phase renforcement est l'équivalent continu tridimensionnel d'un réseau d'inclusions, vues sous l'angle de " poutres $)$.

\section{1}

\section{Contraintes et équilibre par phase}

L'une des caractéristiques majeures de la modélisation biphasique d'un sol renforcé, est de définir les efforts intérieurs et extérieurs, ainsi que d'exprimer l'équilibre, pour chaque phase séparément. Le massif renforcé de la figure 2 est ainsi modélisé par deux systèmes disjoints (mais géométriquement superposés) (Fig. 5) :

- le premier système constitué de la phase matrice relative à la couche renforcée surmontée du matelas granulaire :

- le second système formé de la phase renforcement représentant le réseau d'inclusions.

En l'absence des forces de pesanteur, l'équation d'équilibre dans la phase renforcement s'écrit :

$$
\frac{d n^{r}}{d z}(z)-I(z)=0
$$

où $\mathrm{n}^{r}$ est la contrainte uniaxiale représentant l'effort axial dans les inclusions de renforcement par unité de surface transversale, tandis que I est une densité volumique d'effort vertical décrivant en tout point, à l'échelle macroscopique du modèle biphasique, l'action par frottement latéral du sol sur les inclusions. De même, l'équation d'équilibre dans la phase matrice, projetée sur la direction verticale, s'écrit :

$$
\frac{\mathrm{d} \sigma_{z i}^{m}}{\mathrm{~d} z}(\mathrm{z})+\mathrm{I}(\mathrm{z})=0
$$

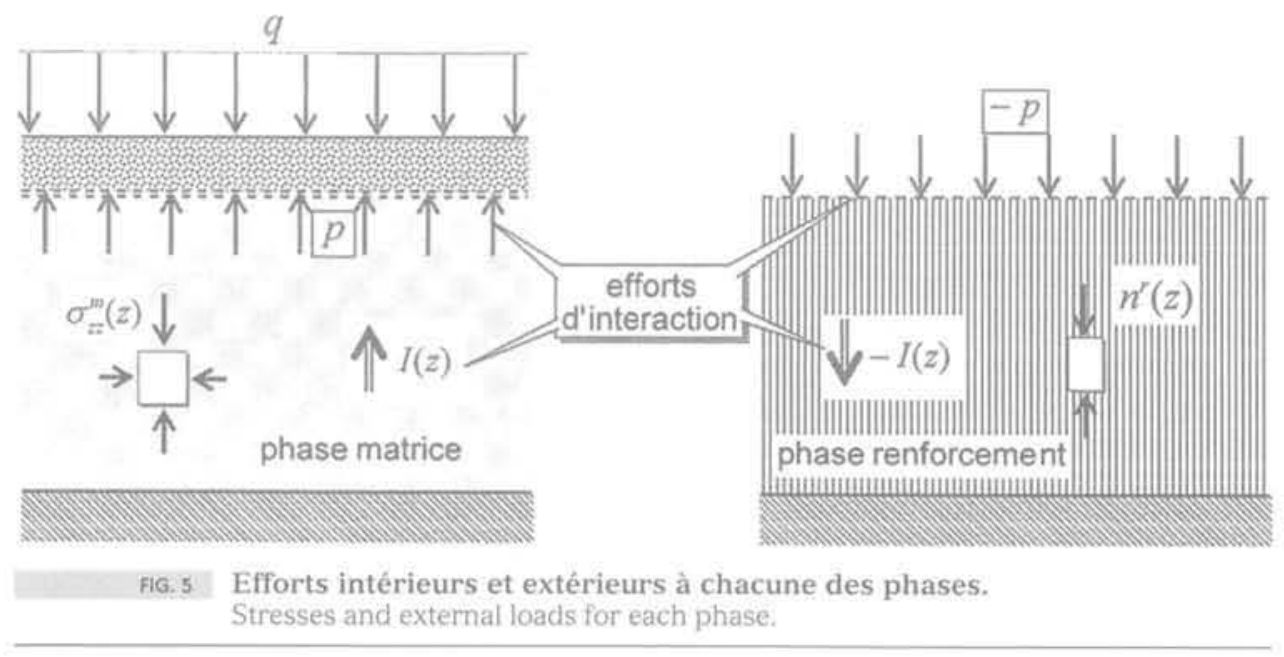


où $\sigma_{z}^{\mathrm{mi}}$ est la contrainte verticale (supposée principale) dans la phase matrice et I est la densité volumique d'effort interaction représentant en tout point l'action de la phase renforcement sur la phase matrice.

Un second type d'effort d'interaction entre les deux phases est à prendre en compte au niveau du plan de supérieur de la couche renforcée où sont localisées les têtes d'inclusions $(z=\mathrm{H})$. Celui-ci est représenté par une densité surfacique, notée p (comme ( pointe $)$ ), qui agit sur la phase matrice, de sorte que l'équation d'équilibre (10) doit être complétée par l'équation suivante :

$$
\sigma_{z z}^{m}\left(\mathrm{z}=\mathrm{H}^{-}\right)-\sigma_{z z}^{m}\left(\mathrm{z}=\mathrm{H}^{-}\right)+\mathrm{p}=0
$$

qui fait apparaitre une discontinuité de la contrainte verticale dans la phase matrice au franchissement du plan $(\mathrm{z}=\mathrm{H})$. En ce qui concerne la phase renforcement, l'existence de cette densité surfacique de forces d'interaction se traduit par la condition aux limites:

$$
n^{\prime}(\mathrm{z}=\mathrm{H})=-\mathrm{p}
$$

\section{2}

\section{Comportement de chaque phase et lois d'interaction}

Désignant respectivement par $m(z)$ et $r(z)$ les déplacements comptés positivement vers le bas (tassements) à la côte $\mathrm{z}$ dans les phases matrice et renforcement (avec bien évidemment $m(0)=r(0)=0$ ), le comportement élastique des phases se traduit par les équations :

$$
\sigma_{z z}^{m}=-E_{m}^{o e d} \frac{d m}{d z} \text { et } n^{r}=-\alpha^{r} \frac{d r}{d z}
$$

où Eoed est le module oedométrique de la phase et $\alpha^{r}$ la raideur des inclusions de renforcement par unité de surface transversale.

De même on peut postuler des lois d'interaction de la forme:

$$
1=-c^{1}(r-m)
$$

pour l'interaction de frottement latéral, oủ cl désigne la raideur correspondante, ainsi que :

$$
\mathrm{p}=-\mathrm{c}^{\mathrm{p}}(\mathrm{r}(\mathrm{H})-\mathrm{m}(\mathrm{H}))
$$

pour l'interaction en pointe des inclusions, avec la raideur associée $\mathrm{CP}^{\mathrm{P}}$.

\section{4}

\section{Mise en œuvre du modèle biphasique}

Le modèle décrit précédemment est maintenant appliqué à la résolution du problème de tassement de la couche de fondation renforcée, schématisé sur la figure 2. La combinaison des équations d'équilibre (9) et (10) et des équations de comportement élastique (13) et (14) conduit au système différentiel suivant dans la zone renforcée $(0 \leq \mathrm{Z} \leq \mathrm{H})$ :

$$
\left\{\begin{array}{l}
E_{m}^{o e d} \frac{d^{2} m}{d z^{2}}+c^{1}(r-m)=0 \\
\alpha^{r} \frac{d^{2} r}{d z^{2}}-c^{1}(r-m)=0
\end{array}\right.
$$

Par ailleurs, la combinaison des équations (12) et (15) ainsi que de la seconde équation de (13) donne:

$$
\alpha^{r} \frac{\mathrm{dr}}{\mathrm{dz}}(\mathrm{H})+\mathrm{c}^{\mathrm{p}}[\mathrm{r}(\mathrm{H})-\mathrm{m}(\mathrm{H})]=0
$$

De même l'équation d'équilibre (11), associée à la première équation de comportement (13), ainsi qu'à (15), et en tenant compte du fait que $\sigma_{z z}^{m}\left(z=H^{+}\right)=-q$, conduit à :

$$
E_{m}^{o e d} \frac{d m}{d z}(H)=q+c^{p}[r(H)-m(H)]
$$

La solution du système différentiel (16), auquel sont associées les équations (17) et (18), ainsi que les conditions aux limites $m(0)=r(0)=0$, s'obtient analytiquement (Cartiaux, 2006). On a ainsi pour les tassements respectifs des phases matrice et renforcement :

$$
\left\{\begin{array}{l}
m(z)=\frac{q}{E_{m}^{o e d}+\alpha^{r}}\left[z+1 \frac{\alpha^{r}}{E_{m}^{o e d}} \frac{\sinh (z / 1)}{\cosh (H / 1)+\kappa \sinh (H / 1)}\right] \\
r(z)=\frac{q}{E_{m}^{\text {oed }}+\alpha^{r}}\left[z-1 \frac{\sinh (z / 1)}{\cosh (H / 1)+\kappa \sinh (H / 1)}\right]
\end{array}\right.
$$

où l est une longueur caractéristique définie par :

$$
I=\sqrt{\frac{\alpha^{r} E_{m}^{\text {oed }}}{c^{1}\left(\alpha^{r}+E_{m}^{\text {oed }}\right)}}
$$

tandis que $\kappa$ est un paramètre sans dimension défini par

$$
\kappa=I c^{p}\left(\frac{\alpha^{r}+E_{m}^{\text {oed }}}{\alpha^{r} E_{m}^{\text {oed }}}\right)
$$

Les expressions de la contrainte verticale dans la phase matrice et de la contrainte axiale dans la phase renforcement sont par ailleurs :

$$
\left\{\begin{array}{l}
\sigma_{z z}^{m}(z)=\frac{-q}{E_{m}^{o o d}+\alpha^{r}}\left[E_{m}^{o e d}+\alpha^{r} \frac{\cosh (z / 1)}{\cosh (H / 1)+\kappa \sinh (H / l)}\right] \\
n^{r}(z)=-\frac{\alpha^{\tau}}{E_{m}^{o e d}+\alpha^{\tau}} q\left[1-\frac{\cosh (z / 1)}{\cosh (H / 1)+\kappa \sinh (H / 1)}\right]
\end{array}\right.
$$

On observe alors immédiatement qu'un tel modèle biphasique constitue une généralisation de la méthode d'homogénéisation, puisque dans le cas où le coefficient d'interaction $c^{\prime}$ tend vers l'infini, c'est-à-dire lorsque les phases matrice et renforcement sont décrites par le méme champ de déplacement (modèle biphasique avec adhérence parfaite), la longueur caractéristique I tend vers zéro, et le tassement du massif en surface vaut

$$
\delta=m(H)=r(H)=\frac{q H}{E_{m}^{\text {od }}+\alpha^{r}}
$$

retrouvant ainsi le même résultat que celui donné par l'équation (5), à la condition d'identifier le module oedométrique vertical de la matrice à celui de sol, et la raideur axiale de la phase renforcement à celle des inclusions de renforcement par unité de surface transversale:

$$
\mathrm{E}_{\mathrm{m}}^{\mathrm{oed}} \equiv \mathrm{E}_{\mathrm{s}}^{\mathrm{oed}}, \alpha^{\mathrm{f}} \equiv \eta \mathrm{E}_{\mathrm{b}}
$$

La figure 6 représente sur un exemple les courbes donnant d'une part le profil des tassements subis respectivement par les phases matrice et renforcement (Fig. 6a), ainsi que les distributions correspondantes des contraintes dans ces mêmes phases (Fig. 6b). Concernant ce dernier point, on retrouve bien notamment l'allure de la variation de la contrainte dans les renforts en fonction de la profondeur, exprimée en terme de facteur de report de charge $\lambda=-n^{r} / q$, qui 

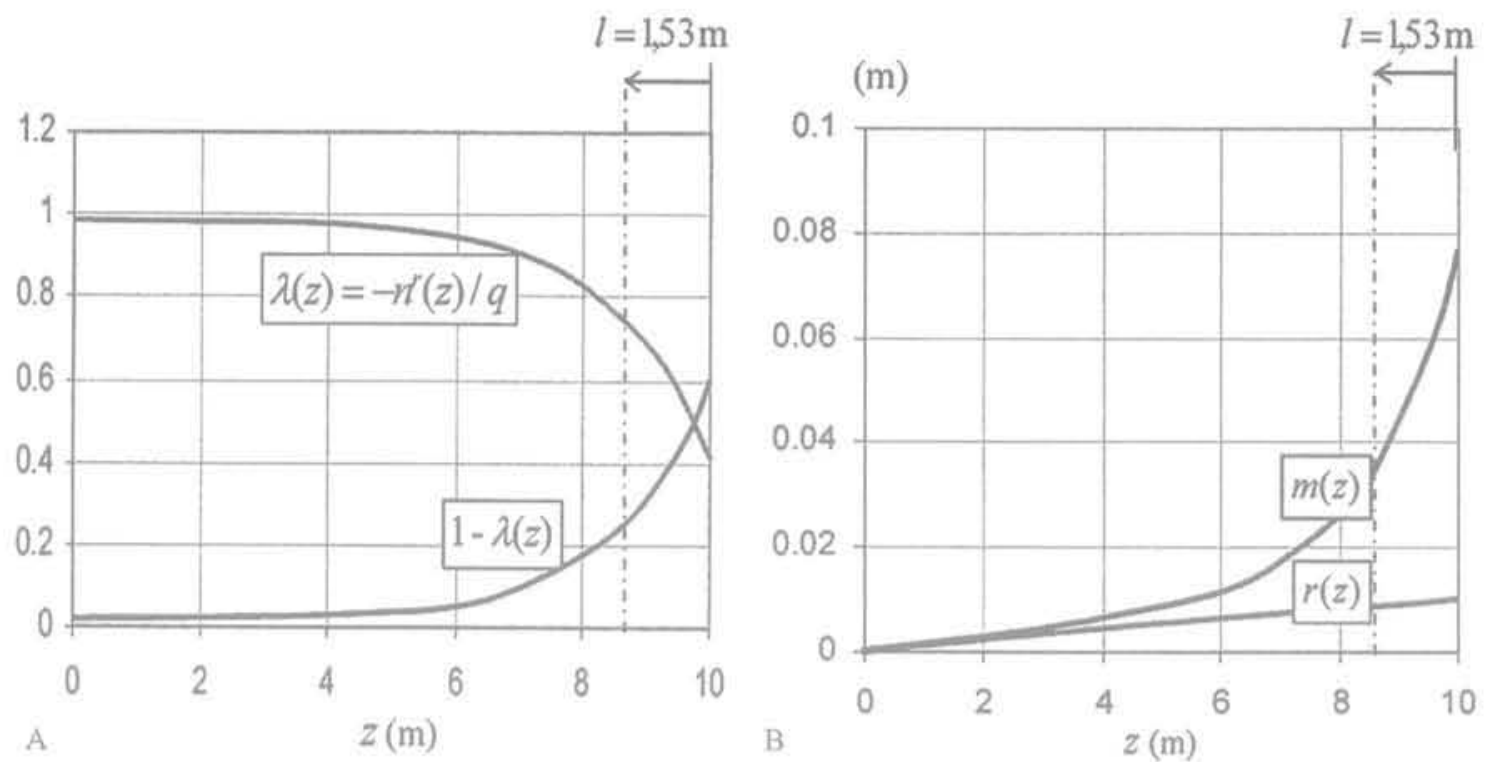

FG. 6 Profils de tassements et de contraintes dans les phases matrice et renforcement. Settlement profiles and stress distributions in the matrix and reinforcement phases,

avait été observée sur la simulation numérique par éléments finis (Fig. 3). On a également reporté sur ces mêmes figures la longueur 1 précédemment introduite (équation (20)), qui peut s'interpréter comme la profondeur caractéristique sur laquelle la solution donnée par le modèle multiphasique s'écarte notablement de celle donnée par la méthode d'homogénéisation.

\section{Identification numérique des paramètres d'interaction du modèle biphasique (Gellée, 2005; Cartiaux, 2006)}

Le point crucial concernant la possibilité de mettre en ceuvre le modèle biphasique reste l'identification de ses paramètres de comportement ( $\left.E_{e d}^{o e d}, a^{r}, c^{d}, c^{p}\right)$ à partir des caractéristiques du sol renforcé. Si un telle identification est immédiate en ce qui concerne les deux premiers paramètres, relatifs au comportement élastique des phases (voir l'équation (24) ci-dessus), il n'en est pas de même pour ce qui est des coefficients d'interaction latérale et de pointe. Nous allons montrer comment une telle identification est réalisable à partir d'un calage de la solution analytique en déplacements donnée par (19) sur les simulations numériques du problème.

Le principe d'une telle identification des paramètres d'interaction est décrit sur la figure 7. Elle consiste à remarquer que la valeur des tassements respectifs des phases matrice et renforcement à la côte z peut être évaluée comme la moyenne calculée sur la section droite de la cellule représentative du sol renforcé, de la distribution des tassements du sol et de l'inclusion de renforcements à cette même côte $z$. Ce que l'on peut écrire :

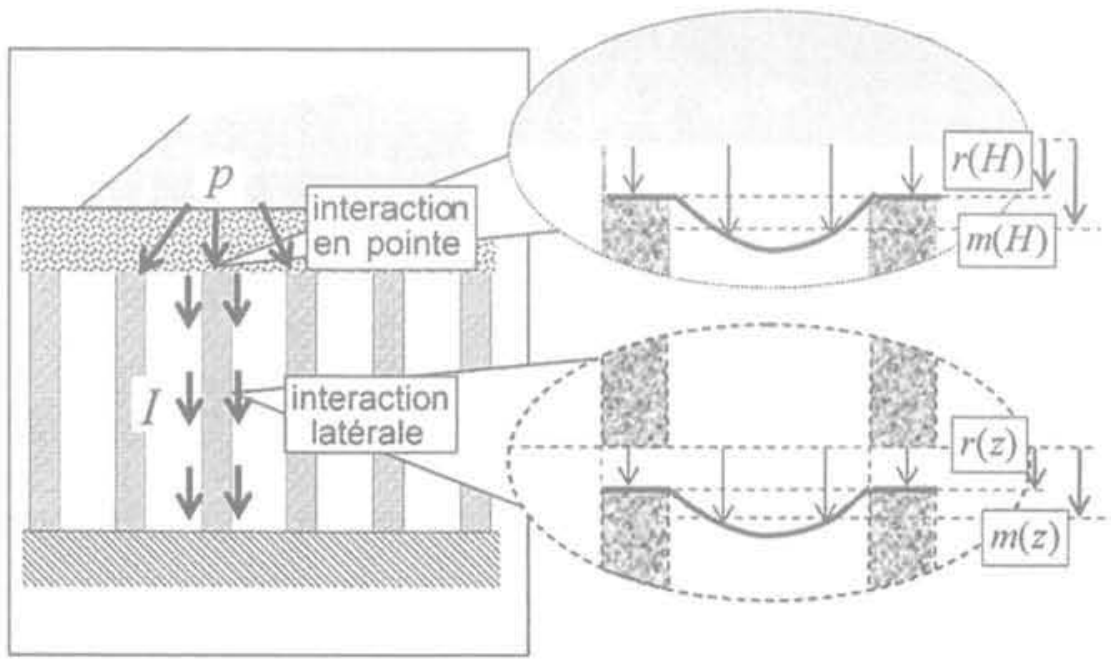

FG.7 Principe de l'identification des coefficients d'interaction du modèle biphasique.

Identification of interaction coefficients for the two-phase model. 
$m(z)=-\frac{1}{\left|A^{s}(z)\right|} \int_{A^{\prime}(z)} \xi_{z}(x, y, z) d x d y, r(z)=$

$-\frac{1}{\left|A^{r}(z)\right|} \int_{A^{\prime}(z)} \xi_{z}(x, y, z) d x d y$

où $\mathrm{A}^{\mathrm{r}}(\mathrm{z})$ et $\mathrm{A}^{\mathrm{s}}(\mathrm{z})$ désignent respectivement les sections transversales à la côte $z$ des parties de la cellule occupées par le sol et l'inclusion. En pratique, cette opération de moyenne des déplacements est réalisée à partir de l'exploitation des résultats des calculs par éléments finis effectuée sur le quart de la cellule élémentaire de sol renforcé, soumise à une sollicitation de compression oedométrique. La figure 8 montre la déformée de cette structure, maillée par des éléments tétraédriques, à l'aide du logiciel Castem, la densité de maillage étant bien évidemment plus forte au voisinage de l'endroit où la tête d'inclusion est en contact avec le matelas granulaire. Dix paliers de mesure sont définis au sein du maillage, plus
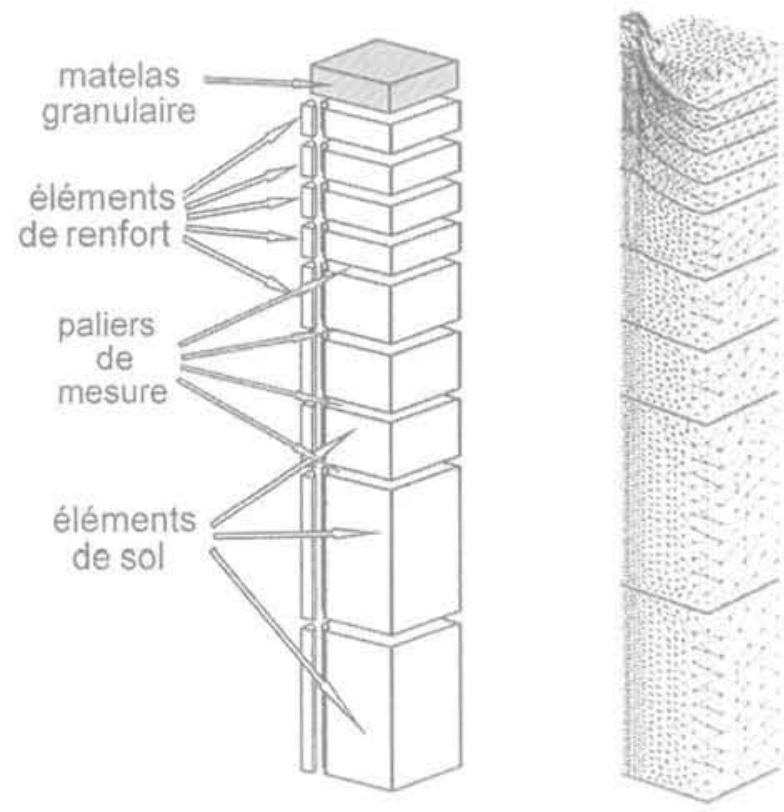

FIG.8 Maillage par éléments finis du quart de la cellule élémentaire de sol renforcé (Cartiaux, 2006).

Finite element mesh of the reinforced soil elementary cell.

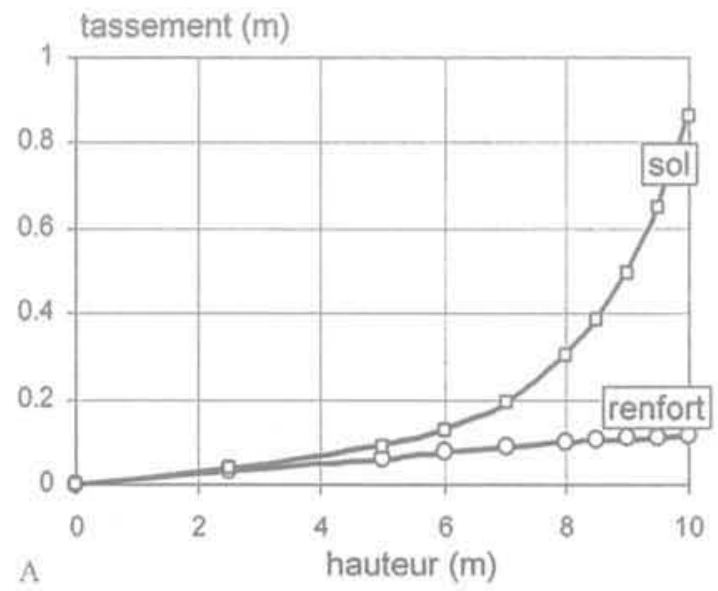

rapprochés en haut qu'en bas de la structure, où sont calculés d'une part la moyenne des tassements dans le sol et l'inclusion par intégration numérique des équation (25), d'autre part les efforts repris respectivement par le sol et par l'inclusion (Gellée, 2005; Cartiaux, 2006).

Il est ainsi possible, pour un jeu de données fixé, de tracer les profils numériques de tassement en fonction de la profondeur, puis d'identifier les paramètres d'interaction par ajustement avec les courbes analytiques correspondantes. La figure 9 illustre une telle procédure appliquée à une configuration type définie par le jeu de paramètres géométriques et mécaniques suivant: $\mathrm{E}_{\mathrm{s}}=10 \mathrm{MPa} ; \mathrm{E}_{\mathrm{b}}=30000 \mathrm{MPa} ; \mathrm{v}_{\mathrm{s}}=0,3 ; \mathrm{H}=10 \mathrm{~m}$ (26) $\eta=0,03 ; \mathrm{e}=2 \mathrm{~m} ; \mathrm{h}=0,5 \mathrm{~m} ; \mathrm{E}_{\mathrm{g}}=30 \mathrm{MPa} ; \mathrm{v}_{\mathrm{g}}=0,3$

La figure $9(\mathrm{a})$ montre les profils de tassements en fonction de la hauteur, déterminés par voie numérique (points) et par voie analytique (courbes en traits pleins), ces dernières étant ajustées en fonction des paramètres d'interaction $c^{\prime}$ et $\mathrm{c}^{\mathrm{p}}$, de façon à rendre minimum l'écart moyen quadratique. On observe un très bon accord entre ces courbes analytiques et les points numériques, qui est confirmé par la figure 9(b), montrant la façon dont se répartit la charge appliquée $(\mathrm{q}=1 \mathrm{MPa})$ entre le sol et les renforts en fonction de la profondeur.

Systématisant une telle démarche, une étude paramétrique a été entreprise (Gellée, 2005), puis prolongée (Cartiaux, 2006), qui a permis d'établir des formules analytiques simples donnant la valeur des paramètres d'interaction du modèle multiphasique en fonction des différents paramètres géométriques et mécaniques qui définissent le problème du tassement d'un couche renforcée soumise à une charge uniforme. Les expressions trouvées sont les suivantes (Cartiaux, 2006) :

- Coefficient d'interaction latérale

$$
c^{i}=c_{0}^{1} \eta\left(\frac{\eta+\eta_{0}^{1}+\eta_{1}^{1}}{\eta+\eta_{1}^{1}}\right)\left(1-2 v_{s} / 3\right) \frac{E_{s}}{e^{2}}
$$

où les paramètres sans dimension valent :

$$
c_{0}^{1}=35,0 ; \eta_{0}^{1}=0,055 ; \eta_{1}^{1}=0,0025
$$

- Coefficient d'interaction en pointe

$$
c^{p}=\eta\left(\frac{\eta+\eta_{0}^{p}+\eta_{1}^{p}}{\eta+\eta_{1}^{p}}\right)\left(\frac{e_{0}}{e}+\frac{h_{0}}{h}\right) E_{g}
$$

avec :

$$
\eta_{0}^{p}=0,025 ; \eta_{1}^{p}=0,0025 ; e_{0}=5 ; h_{0}=0,7
$$

\section{contraintes $(\mathrm{MPa})$}

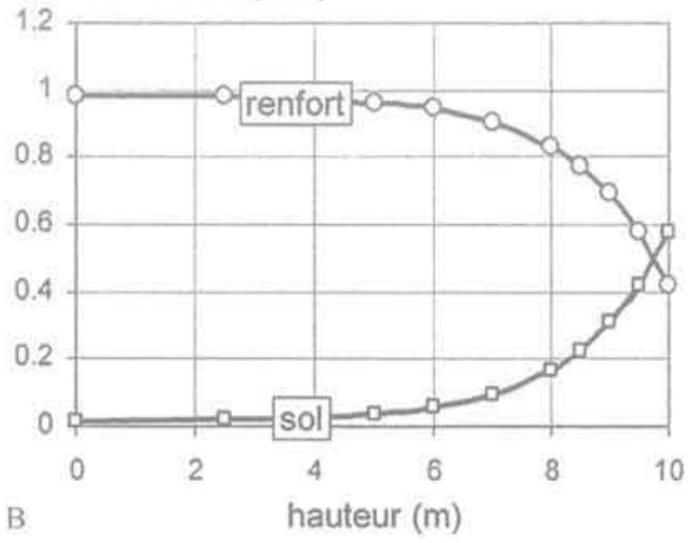

FIG. 9 Calage des courbes analytiques (traits pleins) et numériques (points). Fitting of analytical (solid lines) and numerical (points) curves. 


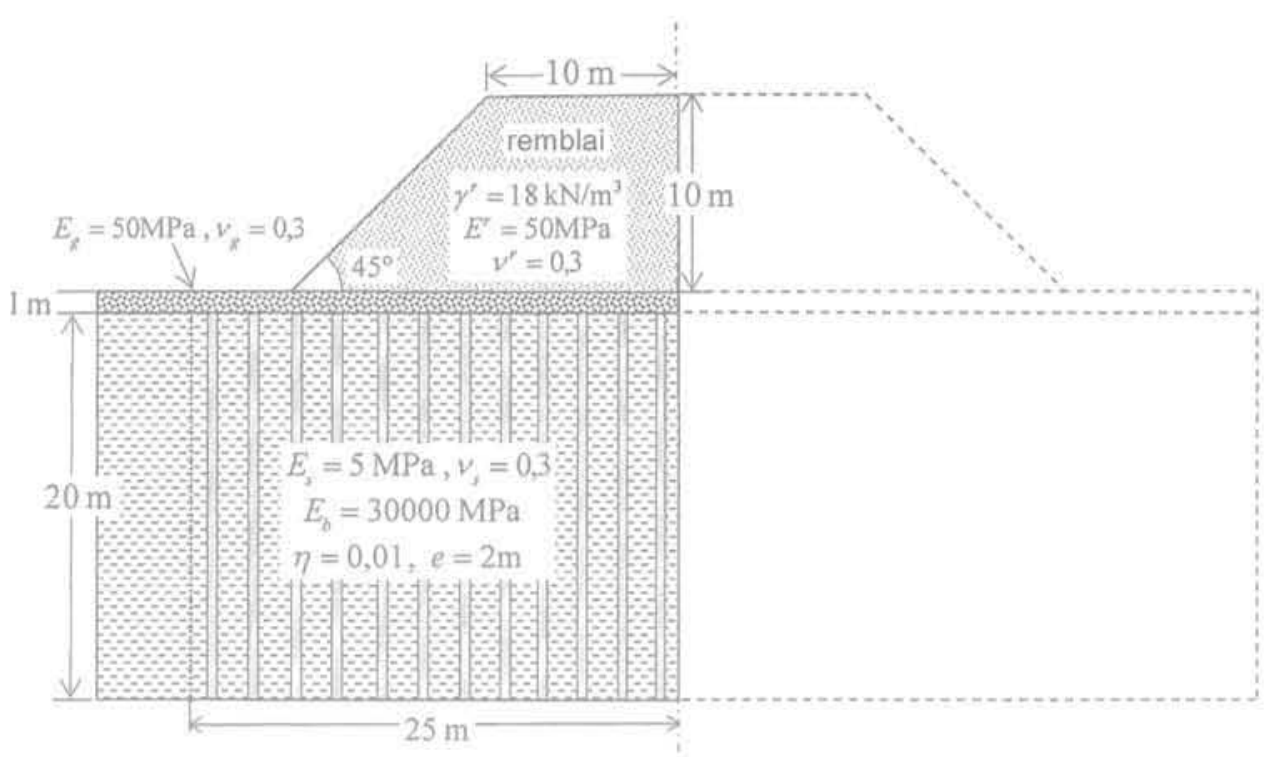

FG. 10 Calcul du tassement d'un sol renforcé par inclusions rigides soumis à l'action du poids d'un remblai.

Settlement analysis of a reinforced soil layer subject to the weight of an embankment.

Il est important de noter que la validité de ces formules est assurée tant que:

$$
\eta<0,2 \text { et } h>0,2 \mathrm{e}
$$

$c^{\prime}$ est-à-dire qu'elles fournissent une évaluation des coefficients d'interaction avec une très bonne précision, puisque l'erreur relative demeure toujours inférieure à $3 \%$. La première condition est en pratique toujours satisfaite, tandis que la seconde exige que le matelas de répartition soit d'une épaisseur suffisante au regard de l'espacement entre les inclusions.

La formule (27a) donnant l'expression du coefficient d'interaction latérale c' montre que, toutes choses étant égales par ailleurs, en particulier à fraction volumique du renforcement $\eta$ fixée, ce coefficient varie en proportion inverse du carré de l'espacement e entre les inclusions. Il en résulte que le fait de resserrer le réseau d'inclusions (en réduisant corrélativement leur diamètre de façon à garder la fraction volumique constante : équation (1)), conduit à une augmentation de ce coefficient, et donc en vertu de la définition (20) à une diminution de la longueur l de la zone d'accrochage où le chargement se transmet progressivement aux inclusions par le biais du frottement latéral.

\section{6}

\section{Un exemple d'application}

L'intérêt principal du modèle multiphasique ici proposé, réside dans le fait, qu'une fois identifiés les paramètres d'interaction grâce aux expressions analytiques (27) et (28) établies sur le problème simplifié d'un massif renforcé d'extension infinie soumis à une surcharge uniforme, il permet de traiter une grande variété d'ouvrages mettant en jeu une technique de renforcement par inclusions rigides, sans restriction particulière concernant la géométrie de la zone renforcée ou le chargement appliqué en surface. Un code de calcul par éléments finis mettant en œuvre une telle modélisation multiphasique a été développé (Hassen et de Buhan, 2005) qui permet la simulation numérique du comportement de ce type d'ouvrage, et notamment le calcul des tassements engendrés par l'application d'un char- gement. La figure 10 donne l'exemple d'un sol de fondation compressible devant supporter la surcharge consécutive à la construction d'un remblai de plate forme en surface. En raison de la symétrie du problème, seule une moitié de l'ouvrage est modélisée en conditions de déformations planes dans le plan transversal à l'axe du remblai. L'ensemble des données géométriques et mécaniques du problème est résumé sur la figure 10. Les effets de cisaillement et de flexion dans les inclusions, susceptibles de se manifester en raison des déplacements latéraux qui apparaissent notamment sur les bords du remblai, sont provisoirement négligés.

Les paramètres à entrer dans le calcul par éléments finis mettant en cuvre le modèle biphasique sont donnés par les équations (24), (27) et (28), soit :

$\alpha^{r}=300 \mathrm{MPa}, \mathrm{c}^{\mathrm{I}}=1,89 \mathrm{MPa} / \mathrm{m}^{2}, \mathrm{c}^{\mathrm{p}}=4,8 \mathrm{MPa} / \mathrm{m}$

La déformée de l'ouvrage sous l'action du chargement représenté par le poids du remblai est représentée sur la partie droite de la figure 11 , tandis que la partie gauche de cette même figure représente la carte des isovaleurs du tassement vertical au sein de l'ouvrage, qui montre clairement, comme l'on pouvait s'y attendre, que le tassement maximal est obtenu sur l'axe de symétrie de l'ouvrage.

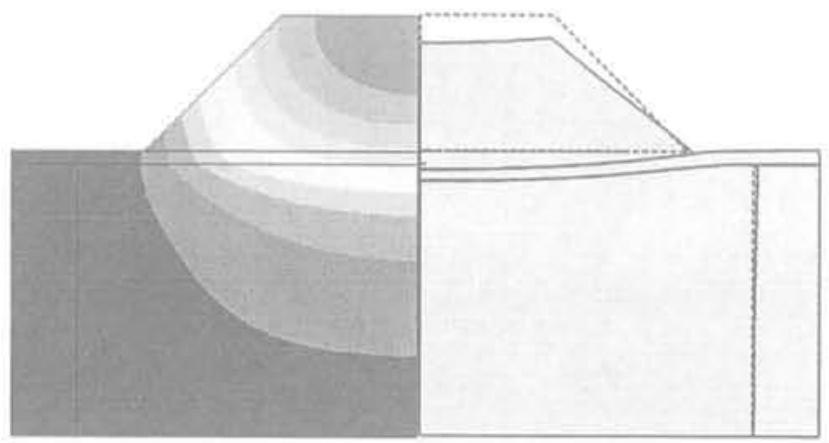

FG.11 Déformée de Youvrage et isovaleurs du tassement.

Deformed configuration and contours of settlements. 


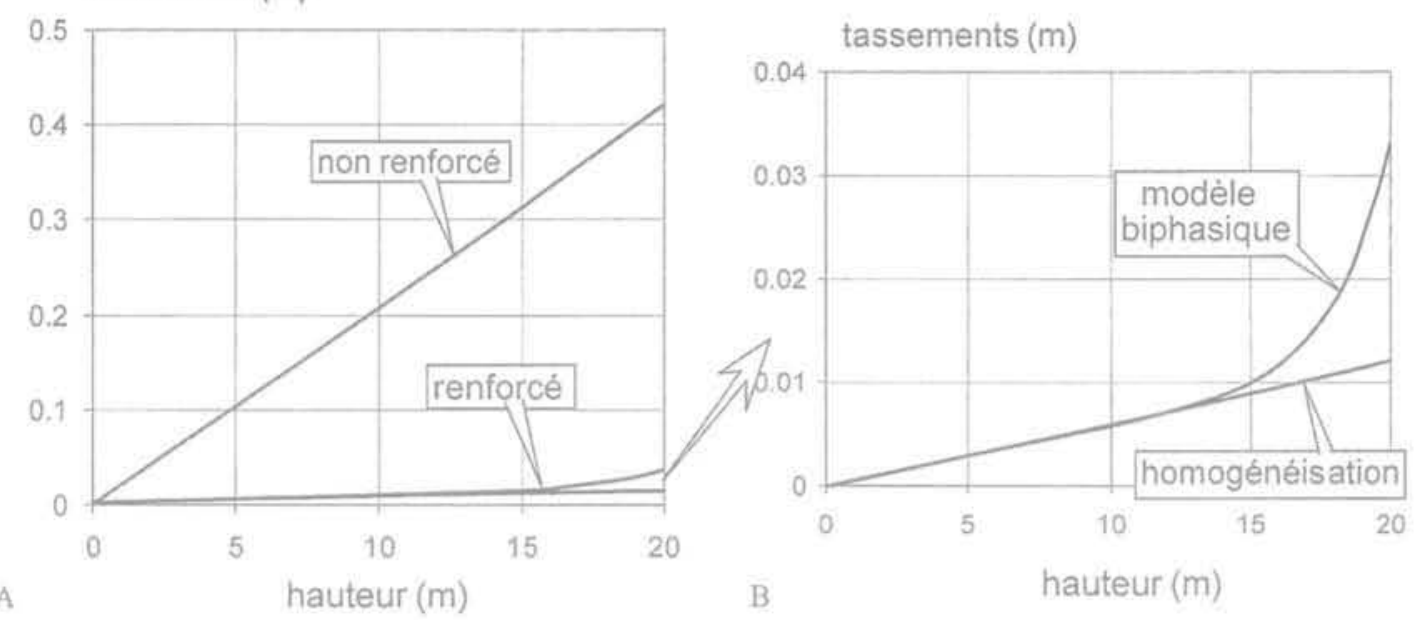

FIG, 12 Profils de tassement dans la couche de sol sur l'axe de symétrie. Settlement profiles along the symmetry axis.

La figure 12 permet de préciser les choses d'un point de vue quantitatif. Elle montre les profils de tassement calculés le long de l'axe de symétrie de l'ouvrage dans les trois situations suivantes:

- cas du sol non renforcé. Le profil est quasi-linéaire, la valeur maximale du tassement en surface étant égale à environ $42 \mathrm{~cm}$;

- cas du sol renforcé, traité par la méthode dhomogénéisation (obtenue à partir du modèle multiphasique en prenant de très grandes valeurs pour les coefficients d'interaction). Le profil est là encore quasi-linéaire, mais la valeur maximale du tassement en surface n'étant plus qu'à peine supérieure à $1 \mathrm{~cm}$ :

- enfin la troisième courbe intermédiaire se réfère au calcul par le modèle multiphasique qui prend en compte les interactions latérale et de pointe par le biais des coefficients $\mathrm{c}^{t}$ et $\mathrm{c}^{\mathrm{p}}$. On observe que la courbe correspondante est confondue avec celle calculée par la méthode d'homogénéisation jusqu'à une hauteur d'environ $13 \mathrm{~m}$, puis qu'elle s'en écarte très sensiblement au fur et à mesure que l'on s'approche de la surface. Le tassement maximal est égal à plus de $3 \mathrm{~cm}$, soit presque trois fois supérieur à celui évalué par la méthode d'homogénéisation. La figure 13 qui représente la distribution d'efforts dans les renforcements, sous la forme de courbes d'isovaleurs, montre qu'une fraction importante de la surcharge (à peu près égale à $18 \mathrm{kN} / \mathrm{m}^{3} \times 10 \mathrm{~m}=180 \mathrm{kPa}$ ) est reprise par les inclusions de renforcement, notamment dans la partie centrale inférieure de la zone renforcée.

\section{7}

\section{Conclusion}

Le modèle multiphasique ici présenté fournit un outil rapide et performant pour le calcul et le dimensionnement des sols de fondation renforcés par inclusions rigides, qui prend explicitement en compte, par le biais de coefficients préalablement identifiés, les mécanismes d'interaction latérale et en pointe des inclusions de renforcement avec le sol environnant. Dans la situation simple de référence d'un sol d'exten- sion horizontale infinie renforcé par une distribution périodique d'inclusions soumis à une surcharge verticale uniforme, on dispose d'ores et déjà de cet outil, qui se réduit à l'application directe de formules analytiques donnant les profils de tassements et d'efforts en fonction de la profondeur. Dans une configuration plus générale telle que celle traitée dans cet article, le modèle multiphasique intégré dans un code de calcul par éléments finis, permet un gain de temps considérable par rapport à une application directe de la méthode des éléments finis, qui aurait conduit à un maillage tridimensionnel extrêmement complexe de la zone renforcée, et par voie de conséquence à des temps de calcul prohibitifs.

Parmi les nombreuses perspectives de prolongement du présent travail, on peut plus particulièrement citer :

- la prise en compte des efforts de cisaillement et de flexion susceptibles de se développer dans les inclu-

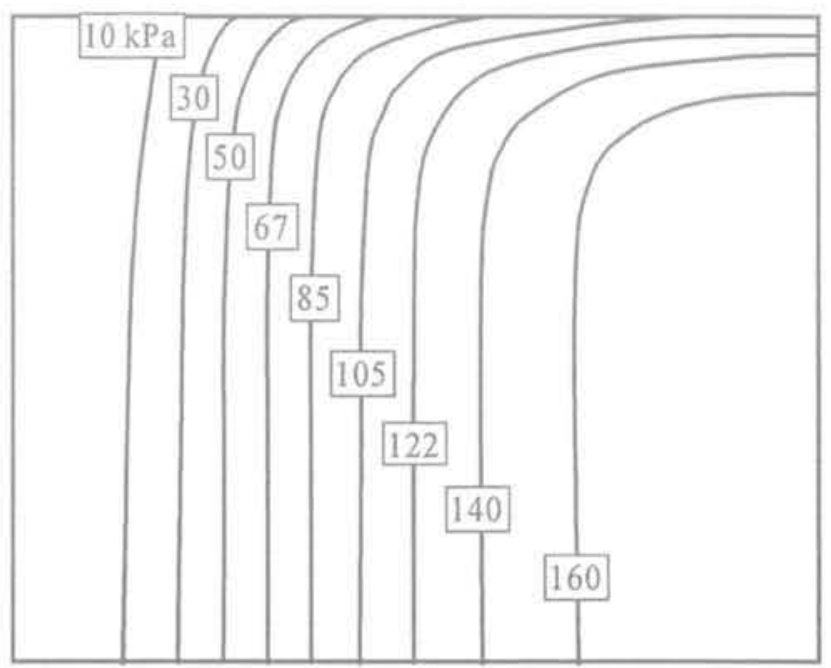

FIG. 13 Isovaleurs de la contrainte de compression dans la phase renforcement.

Contours of compressive stress in the reinforcements. 
sions de renforcement lorsque le chargement comporte une composante latérale (cas du remblai ici traité ou bien encore de sollicitations de type sismique : Hassen et de Buhan, 2005). Il conviendra alors de tenir compte non seulement des interactions longitudinales (c'est-àdire parallèles aux inclusions de renforcement), comme nous l'avons fait ici, mais également d'interactions transversales, analogues à celles décrites pour les pieux de fondations profondes par les courbes « p-y ) (Frank, 1995), de même que les interactions longitudinales renvoient aux courbes $(t-z)$;
- l'identification de lois d'interaction de type élastoplastique offrant une description plus réaliste du comportement des matériaux, notamment au voisinage des têtes d'inclusions où les concentrations de contrainte laissent à penser qu'aussi bien le matelas granulaire que le sol sousjacent sont sollicités bien au-delà de leur domaine d'élasticité. On pourra se limiter, au moins dans un premier temps, à des lois élastiques parfaitement plastiques (Bennis et de Buhan, 2003), pour lesquelles il s'agira d'identifier par le même type de procédure que celle utilisée dans cet article pour l'élasticité, les seuils de plasticité parfaite.

\section{Bibliographie}

Alexiew D., Vogel W, - Remblais ferroviaires renforcés sur pieux en Allemagne : projets phares. Travaux, 786 2002, p. 47-52.

Bennis M. Buhan (de) P. - A multiphase constitutive model of reinforced soils accounting for soil-inclusion interaction behaviour. Math. Comput. Modelling, 37 , 2003, p. 469-475.

Bernaud D., Buhan (de) P., Maghous S. Calcul numérique des tunnels boulonnés par une méthode d'homogénéisation. Revue française de géotechnique, 73, 1995, p. 53-65.

Briançon L, - Renforcement des sols pa: inclusions rígides: état de l'art en France et à l'étranger. IREX, Paris, 2003, 185 p.

Briançon L., Kastner R., Simon B., Dias D,État des connaissances : amélioration des sols par inclusions rigides. Symposium International ASEP-GI, Dhouib et al. (ss la dir.), 2004, p. 15-43.

Buhan (de) P, Sudret B. - Micropolar multiphase model for materials reinforced by linear inclusions. Eur. J. Mech A/Solids, 19, 2000, p. 669-687.

Cartiaux F.-B. - Contribution au développement d'un modèle de calcul multiphasique des ouvrages renforcés par inclusions riaides. Mémoire de stage d’option scientifique, École polytechnique, 2006, 69 p.
Combarieu O, - Amélioration des sols par inclusions rigides verticales. Application à l'édification de remblais sur sols médiocres. Revue française de géotechnique, 44, 1988, p. 57-79.

Gellée A. - Contribution au calcul des sols renforcés par inclusions rigides. Mémoire de stage scientifique, ENPC. 2005, $67 \mathrm{p}$.

Greuell E., Buhan (de) P., Panet M. Salençon J.- Behaviour of tunnels reinforced by untensioned bolts. 13th ICSMFE, New-Dehli, 1994, p. 869-872.

Hassen G. Buhan (de) P. - A two-phase model and related numerical tool for the design of soil structures reinforced by stiff linear inclusions. Eur. J. Mech. A/Solids, 24, 2005, p. 987-1001.

Hassen G. Buhan (de) P. - Elastoplastic multiphase model for simulating the response of piled raft foundations subject to combined loadings. Int. J. Num. An. Meth. Geomech., 30, 2006, p. 843 864

Jenck O., Dias D., Kastner R. - Soft ground improvement by vertical rigid piles. Two-dimensional physical modelling and comparison with current design methods. Soils and Foundations, vol. 45 , $\mathrm{n}^{\circ} 6.2005$, p. $15-30$.
Lacazedieu M., Plomteux C., Corbet S. Shaw-Smith E. - Usage intensif de CMC pour l'amélioration de sol sur la « Newport Southern Distributor Road 2 (UK). $16^{\circ}$ ICSM GE, Osaka, 2004, p. 12151218

Liausu Ph., Pezot B. - Renforcement des sols mous par colonnes à module controlè, $15^{\bullet}$ ICSMGE, 2001, Istanbul,

Pecker A. - Capacity design principles for shallow foundations in seismic areas. Keynote lecture, 11th Eur. Conf. Earthquake Eng., 1998, Paris.

Pecker A. Teyssandier J.-P. - Seismic design for the foundations of the RionAntirion bridge. Proc. ICE, Geotechnical Engineering, 131. 1998, Paper number 13311, p. 4-11.

Rospars C. Bourgeois E., Humbert P. Buhan (de) P. - Un modèle de calcul simplifié pour l'évaluation du tassement d'un massif de fondation renforcé par inclusions rigides. $10^{\circ}$ ICSMGE, Osaka, 2005, p. 855-858.

Sudret B., Buhan (de) P. - Multiphase model for inclusion-reinforced geostructures: Application to rockbolted tunnels and piled raft foundations. Int. J. Num. An. Meth. Geomech., 25, 2001. p. 155-182. 